\title{
Unsteady flow due to a disk executing non-torsional oscillation and a Newtonian fluid at infinity rotating about non-coaxial axes
}

\author{
H VOLKAN ERSOY \\ Department of Mechanical Engineering, Yildiz Technical University, 34349 Istanbul, Turkey \\ e-mail: hversoy@yildiz.edu.tr
}

MS received 8 October 2015; revised 15 June 2016; accepted 14 September 2016

\begin{abstract}
This paper is concerned with the unsteady flow of a disk performing non-torsional oscillation in its own plane and a Newtonian fluid at infinity while they are initially rotating with the same angular velocity about non-coaxial axes. For a more general study, it is considered that the disk executes non-torsional oscillation along any desired direction in its own plane. An exact solution obtained for the velocity field is compared with a periodic solution presented in order to find the time when the periodic flow starts. A very good agreement is found between the two solutions in the periodic state. It is an interesting result that the $x$ - and $y$-components of the force per unit area exerted by the fluid on the disk vary in almost opposite direction when the non-torsional oscillation takes place along the eccentricity direction. Further, the change in the $y$-component of the translational velocity becomes noticeable in this case.
\end{abstract}

Keywords. Newtonian fluid; oscillating disk; non-coaxial rotation; exact solution; periodic flow.

\section{Introduction}

The steady flow induced by the non-coaxial rotation of a disk and a Newtonian fluid at infinity was first studied by Coirier [1]. Kasiviswanathan and Rao [2] were the first to examine the periodic flow due to a disk executing nontorsional oscillations in its own plane and the fluid at infinity rotating with the same angular velocity about distinct axes. They found an exact solution for the velocity field in the case of a porous disk. The same problem was generalized by Hayat et al [3] and Guria et al [4]. Guria et al [4] also presented a solution for the shear stresses on the disk.

Erdoğan [5] studied the unsteady flow due to the noncoaxial rotation of a disk executing non-torsional oscillations in its own plane and a Newtonian fluid at infinity while they are initially rotating about a common axis. He obtained an exact solution of the velocity field for all times. This problem was extended to the MHD flow for a porous disk by Hayat et al [6]. Hayat et al [7] investigated the problem of Hayat et al [6] for the generalized non-torsional oscillations. Hayat et al [8] examined the Hall current effect as well as suction and blowing effects. The unsteady flow produced by the rotation of a disk and a non-Newtonian fluid at infinity about distinct axes was first investigated by Hayat et al [9]. They examined the problem in the case of a porous disk for a second-grade fluid with small material modulus. Hayat et al [10] extended this problem to the MHD flow. Hayat et al [6-10] also presented the asymptotic solutions for large times. Wang and $\mathrm{Wu}$ [11] studied the MHD flow of a third-order fluid for a porous disk and obtained a numerical solution for both the velocity field and the shear stresses in the fluid. They also considered a slip boundary condition at the disk. Erdoğan [5], Hayat et al [6-10] and Wang and $\mathrm{Wu}[11]$ took into account that the disk starts to rotate about another parallel axis and to perform non-torsional oscillation while the disk and the fluid at infinity are rotating about a common axis. Further, they considered that the nontorsional oscillation of the disk is in the direction perpendicular to the eccentricity.

This paper studies the unsteady flow caused by a disk executing non-torsional oscillations in the own plane while the disk and a Newtonian fluid at infinity are initially rotating about non-coaxial axes. Thus, the initial condition is the solution presented by Coirier [1]. The oscillation velocity of the disk has two components both in the eccentricity direction and in the direction perpendicular to the eccentricity. Both an exact solution and another solution corresponding to the periodic flow are obtained for the velocity field and the shear stresses in the fluid. Comparison between the two solutions shows a very good agreement for sufficiently large times. The effects of ratio of the frequency of oscillation to the angular velocity of the disk $(k)$, the dimensionless velocity amplitudes in the $x$ - and $y$-directions $\left(V_{x}, V_{y}\right)$ and the dimensionless time $(\tau)$ on the velocity field and the shear stresses that represent the components of the horizontal force per unit area exerted by the fluid on the disk are discussed by plotting graphs. 


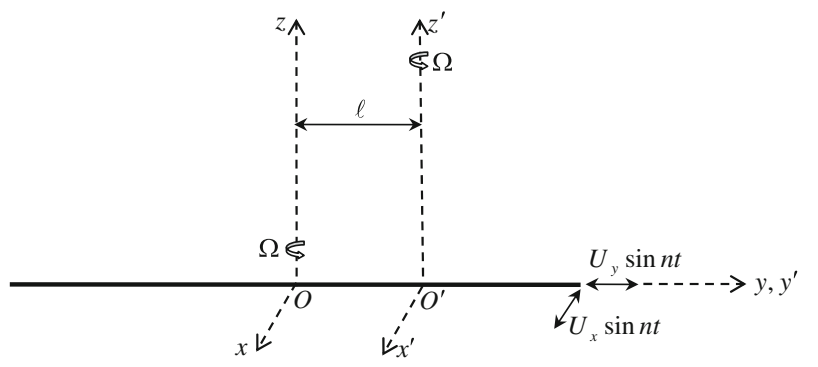

Figure 1. Flow geometry.

\section{Basic equations and solution}

Let us consider an incompressible Newtonian fluid in a semi-infinite domain $z>0$ bounded by an infinite disk at $z=0$. The disk and the fluid at infinity are rotating with the same angular velocity $\Omega$ about two parallel axes normal to the disk at the time $t=0$. The axes of rotation of the disk and the fluid at infinity pass through the origin and a point defined by $x=0$ and $y=\ell$, respectively. In addition to its rotation about the $z$-axis, the disk starts to perform nontorsional oscillation in its own plane with the velocity $\mathbf{U}=\left(U_{x} \mathbf{i}+U_{y} \mathbf{j}\right) \sin n t$. Here, $n$ is the frequency of the

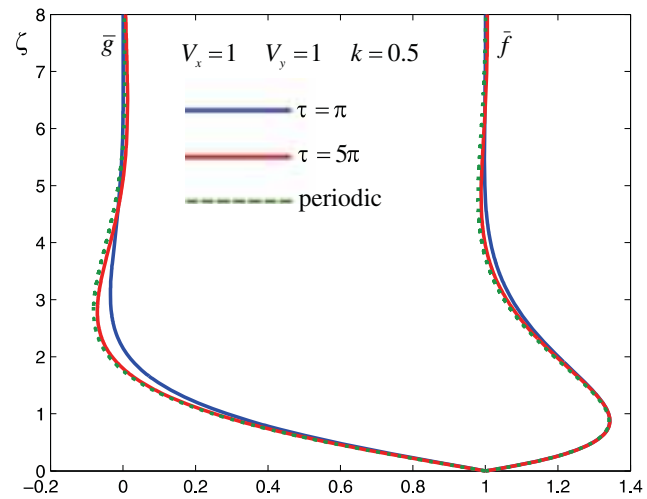

(a)

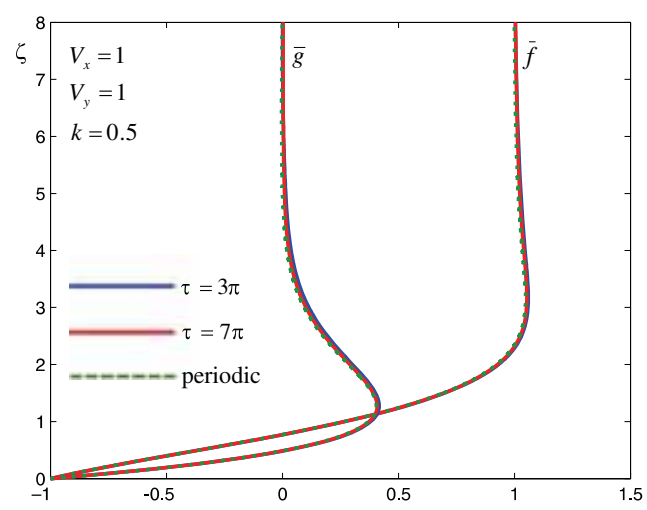

(c) oscillation. The geometry of problem is shown in figure 1 . Therefore, the initial and boundary conditions are

$$
\begin{aligned}
& u=-\Omega y+\hat{f}(z), \quad v=\Omega x+\hat{g}(z), \quad \text { at } t=0 \quad \text { for } z \geq 0, \\
& u=-\Omega y+U_{x} \sin n t, \quad v=\Omega x+U_{y} \sin n t, \quad \text { at } z=0 \\
& \text { for } t \geq 0 \text {, }
\end{aligned}
$$

$$
u=-\Omega(y-\ell), \quad v=\Omega x, \quad \text { at } z \rightarrow \infty \quad \text { for } t \geq 0,
$$

where $u$ and $v$ denote the velocity components in the $x$ - and $y$-directions. The functions $\hat{f}(z)$ and $\hat{g}(z)$, obtained by [1], represent the translational velocity components corresponding to the steady flow induced by the non-coaxial rotation of a disk and a Newtonian fluid at infinity, and are given by

$$
\hat{f}(z)+i \hat{g}(z)=\Omega \ell\left[1-\exp \left(-\sqrt{\frac{\rho \Omega}{2 \mu}}(1+i) z\right)\right],
$$

where $\rho$ and $\mu$ are the density and dynamic viscosity of the fluid, respectively. In the light of these conditions, we seek a solution of the form

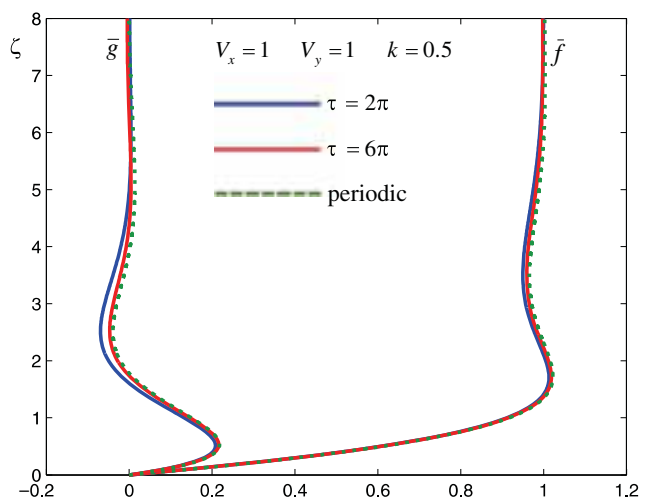

(b)

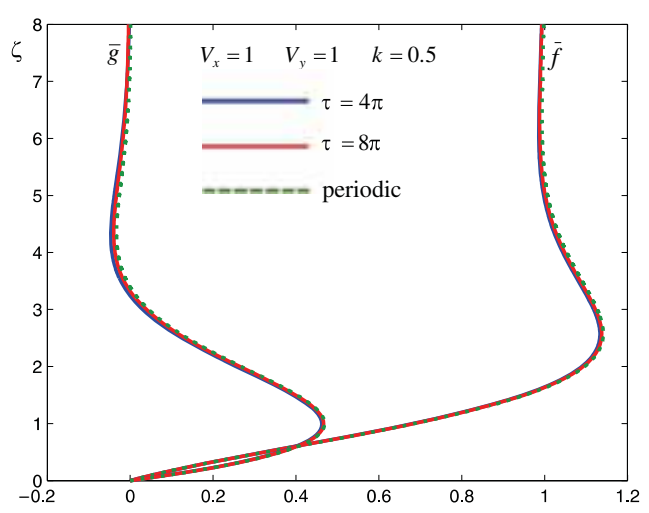

(d)

Figure 2. Transition to periodic flow for $V_{x}=1, V_{y}=1, k=0.5$. 


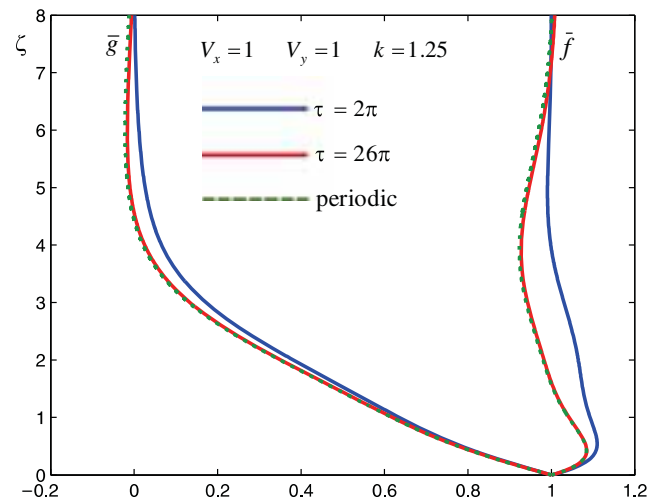

(a)

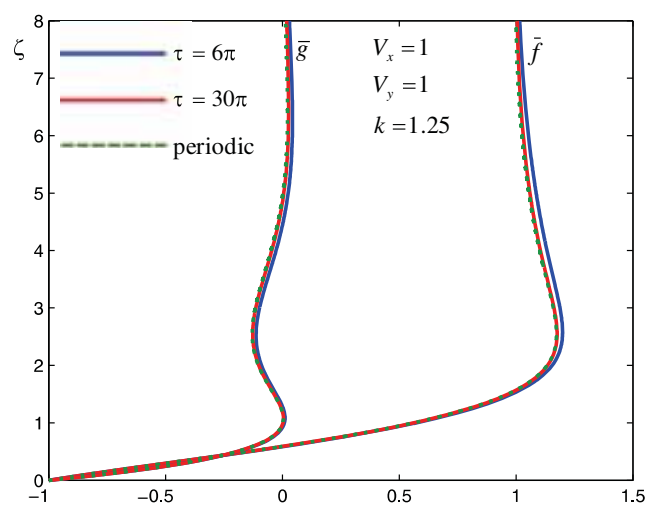

(c)

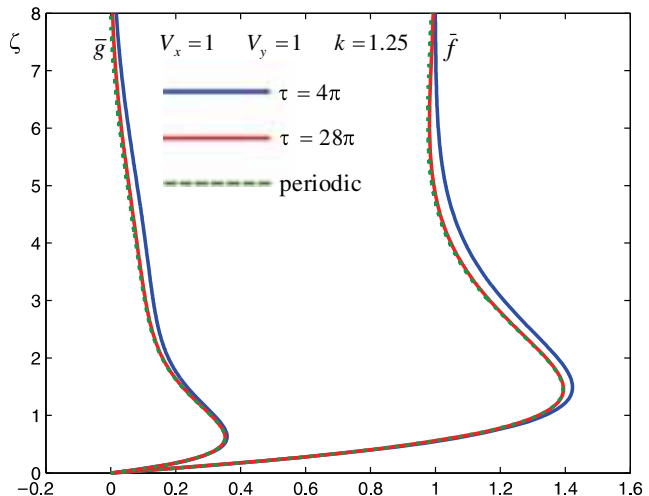

(b)

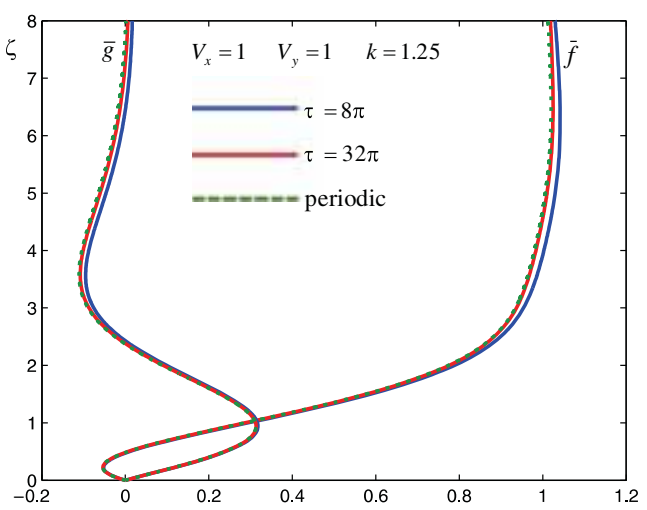

(d)

Figure 3. Transition to periodic flow for $V_{x}=1, V_{y}=1, k=1.25$.

$$
u=-\Omega y+f(z, t), \quad v=\Omega x+g(z, t),
$$

where $f(z, t)$ and $g(z, t)$ denote the $x$ - and $y$-components of the time-dependent translational velocity, respectively. Substituting Eq. (5) into the Navier-Stokes equation, one obtains

$\mu \frac{\partial^{2} f}{\partial z^{2}}-\rho \frac{\partial f}{\partial t}+\rho \Omega g=C_{1}(t), \mu \frac{\partial^{2} g}{\partial z^{2}}-\rho \frac{\partial g}{\partial t}-\rho \Omega f=C_{2}(t)$

Since the fluid at infinity has no shear stress, we find that $C_{1}(t)=0$ and $C_{2}(t)=-\rho \Omega^{2} \ell$ with the help of Eq. (3). If we introduce the dimensionless variables

$$
\begin{aligned}
& \bar{f}(\zeta, \tau)=\frac{f(z, t)}{\Omega \ell}, \bar{g}(\zeta, \tau)=\frac{g(z, t)}{\Omega \ell}, \\
& \tilde{F}(\zeta, \tau)=\bar{f}(\zeta, \tau)+i \bar{g}(\zeta, \tau)-1, \\
& \zeta=\sqrt{\frac{\rho \Omega}{2 \mu}} z, \tau=\Omega t, k=\frac{n}{\Omega}, V_{x}=\frac{U_{x}}{\Omega \ell}, V_{y}=\frac{U_{y}}{\Omega \ell},
\end{aligned}
$$

the governing equation in dimensionless form and the corresponding initial and boundary conditions become

$$
\begin{gathered}
\frac{\partial^{2} \tilde{F}}{\partial \zeta^{2}}-2 \frac{\partial \tilde{F}}{\partial \tau}-2 i \tilde{F}=0 \\
\tilde{F}(\zeta, 0)=-\exp [-(1+i) \zeta] \quad \text { for } \zeta \geq 0, \\
\tilde{F}(0, \tau)=V_{x} \sin k \tau+i V_{y} \sin k \tau-1 \quad \text { for } \tau \geq 0, \\
\tilde{F}(\infty, \tau)=0 \quad \text { for } \tau \geq 0
\end{gathered}
$$

Setting

$$
\tilde{F}(\zeta, \tau)=H(\zeta, \tau) \exp (-i \tau)
$$

we have

$$
\begin{gathered}
\frac{\partial^{2} H}{\partial \zeta^{2}}-2 \frac{\partial H}{\partial \tau}=0 \\
H(\zeta, 0)=-\exp [-(1+i) \zeta] \quad \text { for } \zeta \geq 0 \\
H(0, \tau)=\left[V_{x} \sin k \tau+i V_{y} \sin k \tau-1\right] \exp (i \tau) \text { for } \tau \geq 0
\end{gathered}
$$

$$
H(\infty, \tau)=0 \quad \text { for } \tau \geq 0
$$




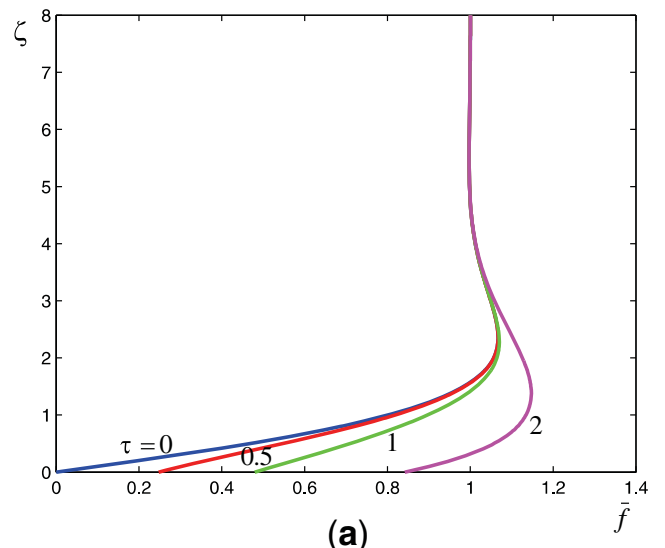

(a)

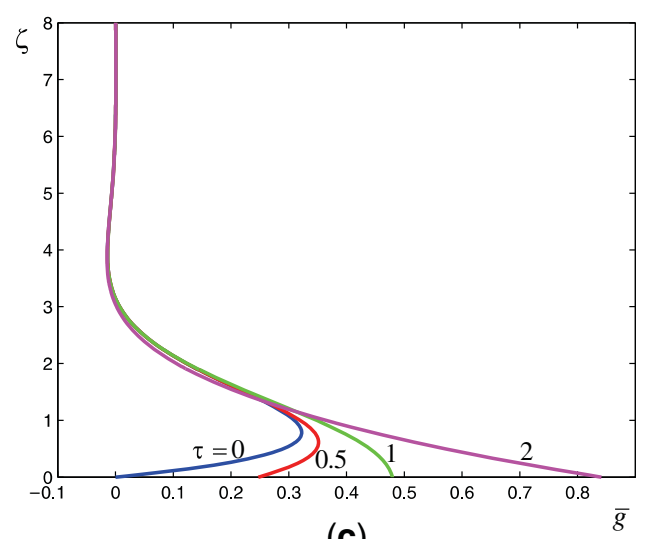

(c)
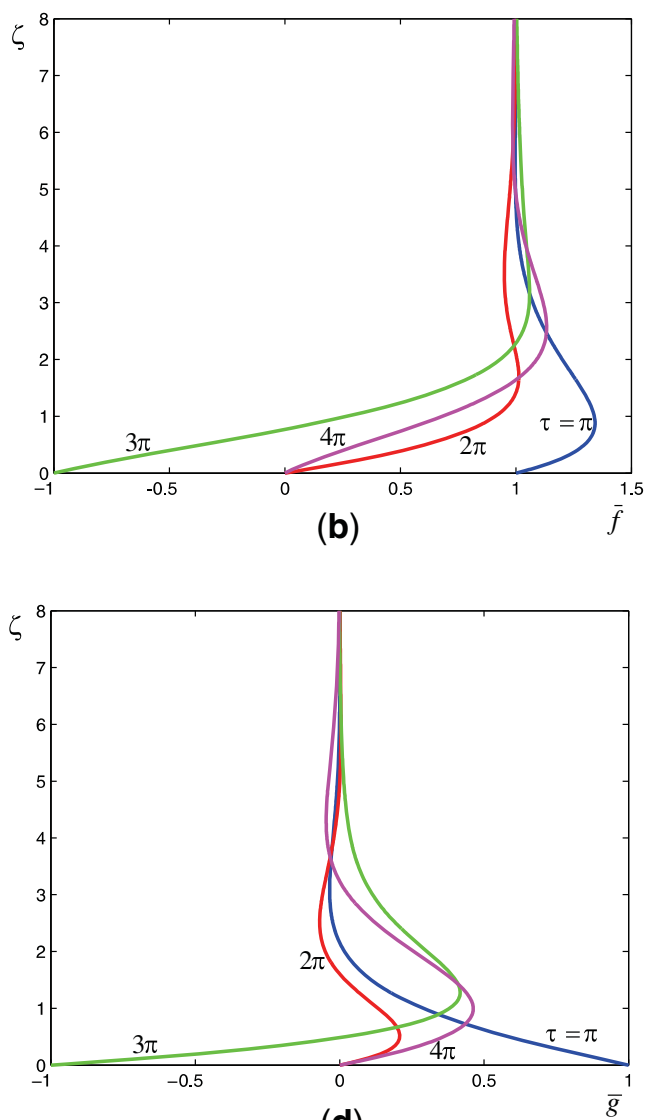

(d)

Figure 4. Variation of $\bar{f}$ and $\bar{g}$ with $\zeta$ for $k=0.5, V_{x}=1, V_{y}=1(\tau=0,0.5,1,2, \pi, 2 \pi, 3 \pi, 4 \pi)$.

The Laplace transform of $H(\zeta, \tau)$ is defined by

$$
\bar{H}(\zeta, s)=\int_{0}^{\infty} H(\zeta, \tau) \exp (-s \tau) d \tau .
$$

Taking the Laplace transforms of Eqs. (13) and (15-16), we get

$$
\begin{gathered}
\bar{H}^{\prime \prime}-2 s \bar{H}=2 \exp [-(1+i) \zeta], \\
\bar{H}(0)=\frac{\left(V_{x}+i V_{y}\right) k}{(s-i)^{2}+k^{2}}-\frac{1}{s-i}, \\
\bar{H}(\infty)=0 .
\end{gathered}
$$

The solution of Eq. (18) satisfying Eqs. (19) and (20) is

$$
\bar{H}=\frac{\left(V_{x}+i V_{y}\right) k}{(s-i)^{2}+k^{2}} \exp (-\sqrt{2 s} \zeta)-\frac{1}{s-i} \exp [-(1+i) \zeta]
$$

The Laplace inversion of Eq. (21) yields

$$
\begin{aligned}
\bar{f}(\zeta, \tau)+i \bar{g}(\zeta, \tau)= & 1-\exp [-(1+i) \zeta] \\
& -\frac{1}{4}\left(V_{y}-i V_{x}\right)[\exp (-i k \tau) A(\zeta, \tau) \\
& -\exp (i k \tau) B(\zeta, \tau)],
\end{aligned}
$$

where

$$
\begin{aligned}
A(\zeta, \tau) & =A_{1}(\zeta, \tau)+A_{2}(\zeta, \tau) \\
A_{1}(\zeta, \tau) & =\exp (a \sqrt{2} \zeta) \operatorname{erfc}\left(\frac{\zeta}{\sqrt{2 \tau}}+a \sqrt{\tau}\right) \\
A_{2}(\zeta, \tau) & =\exp (-a \sqrt{2} \zeta) \operatorname{erfc}\left(\frac{\zeta}{\sqrt{2 \tau}}-a \sqrt{\tau}\right) \\
B(\zeta, \tau) & =B_{1}(\zeta, \tau)+B_{2}(\zeta, \tau) \\
B_{1}(\zeta, \tau) & =\exp (b \sqrt{2} \zeta) \operatorname{erfc}\left(\frac{\zeta}{\sqrt{2 \tau}}+b \sqrt{\tau}\right) \\
B_{2}(\zeta, \tau) & =\exp (-b \sqrt{2} \zeta) \operatorname{erfc}\left(\frac{\zeta}{\sqrt{2 \tau}}-b \sqrt{\tau}\right), \\
a & =(-1+i) \sqrt{\frac{k-1}{2}} \\
b & =(1+i) \sqrt{\frac{k+1}{2}}
\end{aligned}
$$




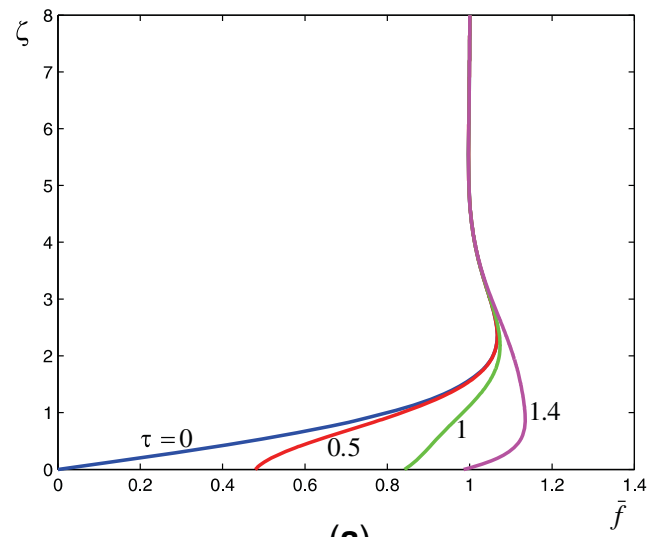

(a)

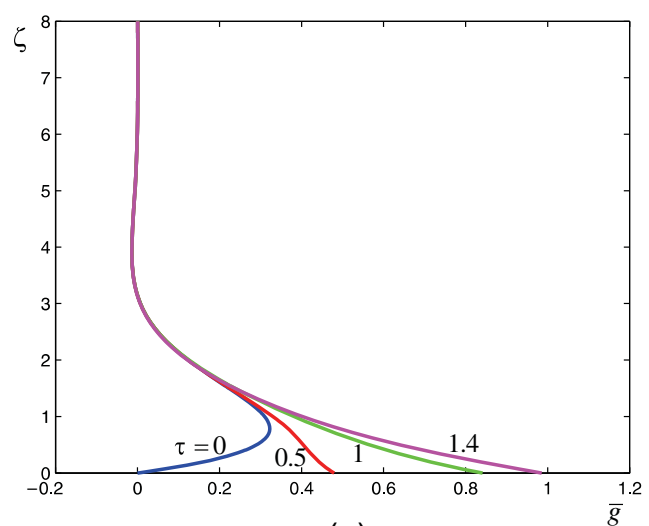

(c)

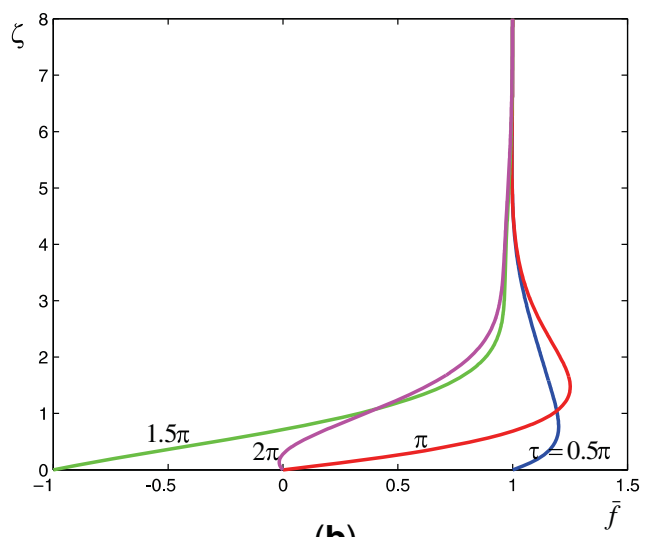

(b)

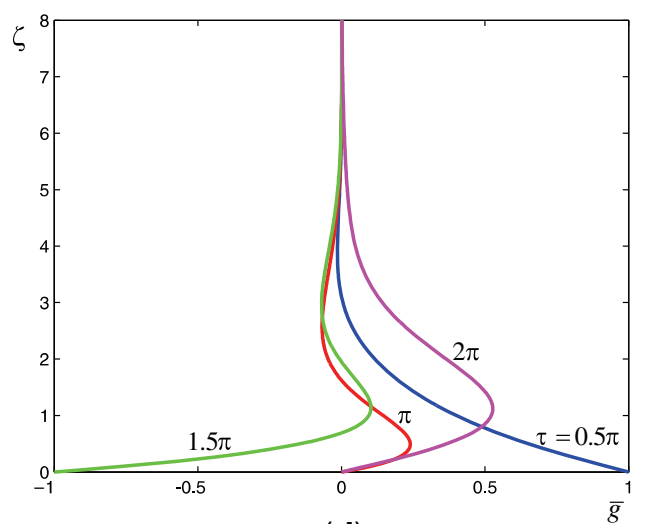

(d)

Figure 5. Variation of $\bar{f}$ and $\bar{g}$ with $\zeta$ for $k=1, V_{x}=1, V_{y}=1(\tau=0,0.5,1,1.4,0.5 \pi, \pi, 1.5 \pi, 2 \pi)$.

The shear stress components $T_{x z}$ and $T_{y z}$ in the fluid are obtained as follows:

$$
\begin{aligned}
\bar{T}_{x z}(\zeta, \tau)+i \bar{T}_{y z}(\zeta, \tau)= & (1+i) \exp [-(1+i) \zeta] \\
& -\frac{1}{4}\left(V_{y}-i V_{x}\right)[\exp (-i k \tau) D(\zeta, \tau) \\
& -\exp (i k \tau) E(\zeta, \tau)]
\end{aligned}
$$

where

$$
\begin{aligned}
\bar{T}_{x z}= & \frac{T_{x z}}{\sqrt{\mu \rho \Omega^{3} / 2} \ell}, \\
\bar{T}_{y z}= & \frac{T_{y z}}{\sqrt{\mu \rho \Omega^{3} / 2 \ell}}, \\
D(\zeta, \tau)= & D_{1}(\zeta, \tau)+D_{2}(\zeta, \tau), \\
D_{1}(\zeta, \tau)= & a \sqrt{2} A_{1}(\zeta, \tau) \\
& -\sqrt{\frac{2}{\pi \tau}} \exp \left[a \sqrt{2} \zeta-\left(\frac{\zeta}{\sqrt{2 \tau}}+a \sqrt{\tau}\right)^{2}\right], \\
D_{2}(\zeta, \tau)= & -a \sqrt{2} A_{2}(\zeta, \tau) \\
& -\sqrt{\frac{2}{\pi \tau}} \exp \left[-a \sqrt{2} \zeta-\left(\frac{\zeta}{\sqrt{2 \tau}}-a \sqrt{\tau}\right)^{2}\right],
\end{aligned}
$$

$$
\begin{aligned}
E(\zeta, \tau)= & E_{1}(\zeta, \tau)+E_{2}(\zeta, \tau) \\
E_{1}(\zeta, \tau)= & b \sqrt{2} B_{1}(\zeta, \tau) \\
& -\sqrt{\frac{2}{\pi \tau}} \exp \left[b \sqrt{2} \zeta-\left(\frac{\zeta}{\sqrt{2 \tau}}+b \sqrt{\tau}\right)^{2}\right], \\
E_{2}(\zeta, \tau)= & -b \sqrt{2} B_{2}(\zeta, \tau) \\
& -\sqrt{\frac{2}{\pi \tau}} \exp \left[-b \sqrt{2} \zeta-\left(\frac{\zeta}{\sqrt{2 \tau}}-b \sqrt{\tau}\right)^{2}\right] .
\end{aligned}
$$

The above solution presented by Eqs. (22)-(25) is valid for all times. An important aim of this paper is to point out the time when the periodic flow starts. For this reason, another solution corresponding to the periodic flow is presented. In this case, it is reasonable to try a solution of the form

$$
\tilde{F}(\zeta, \tau)=\tilde{F}_{0}(\zeta)+\tilde{F}_{1}(\zeta) \cos k \tau+\tilde{F}_{2}(\zeta) \sin k \tau
$$

where $\tilde{F}_{0}(z)$ corresponds to the case of $n=0$. Substituting Eq. (26) into Eq. (8), we get

$$
\begin{gathered}
\tilde{F}_{0}^{\prime \prime}-2 i \tilde{F}_{0}=0, \\
\tilde{F}_{1}^{\prime \prime}-2 i \tilde{F}_{1}-2 k \tilde{F}_{2}=0,
\end{gathered}
$$




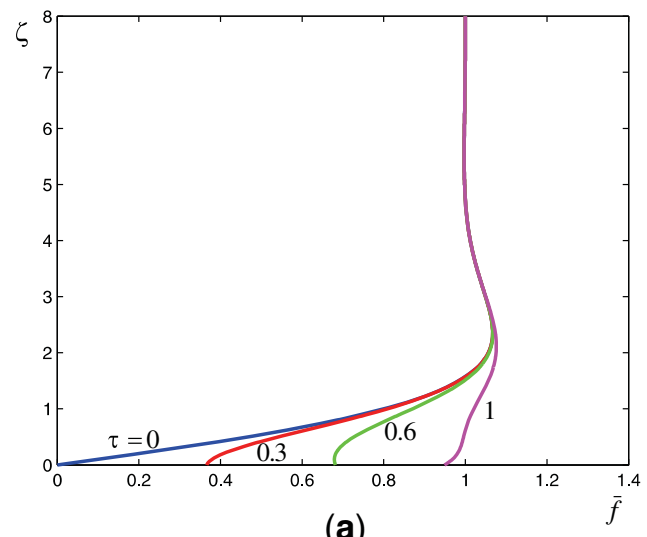

(a)

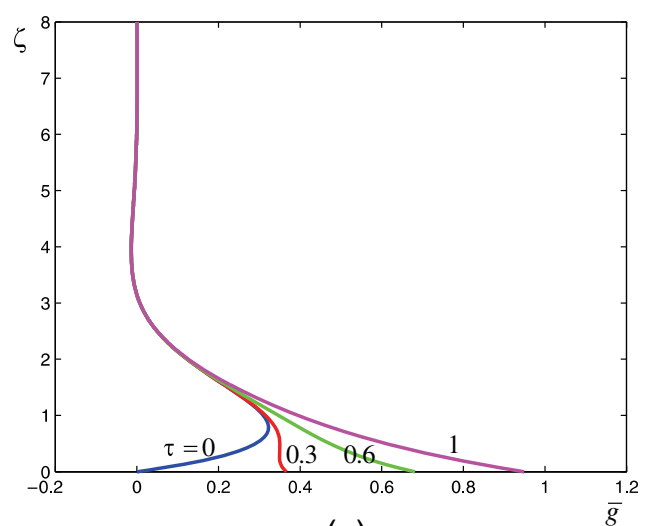

(c)

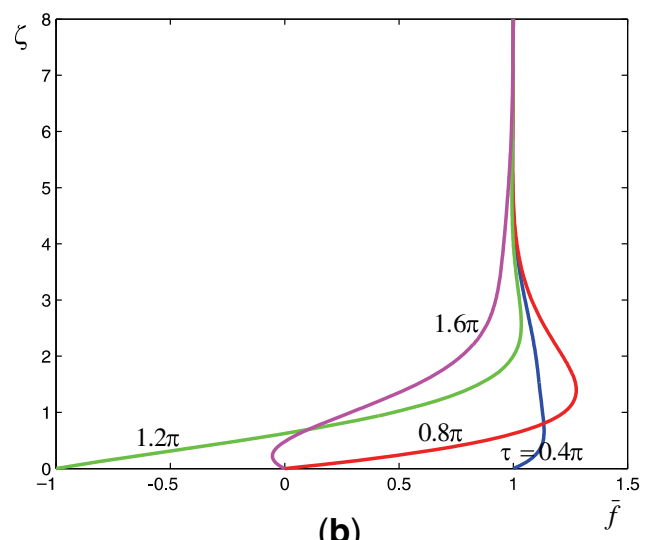

(b)

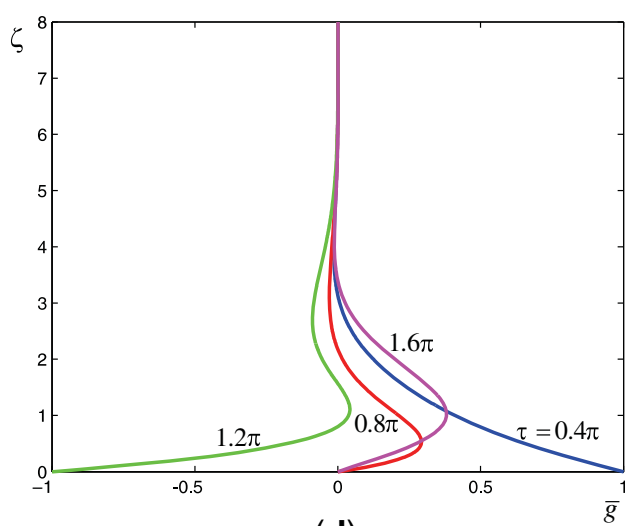

(d)

Figure 6. Variation of $\bar{f}$ and $\bar{g}$ with $\zeta$ for $k=1.25, V_{x}=1, V_{y}=1(\tau=0,0.3,0.6,1,0.4 \pi, 0.8 \pi, 1.2 \pi, 1.6 \pi)$.

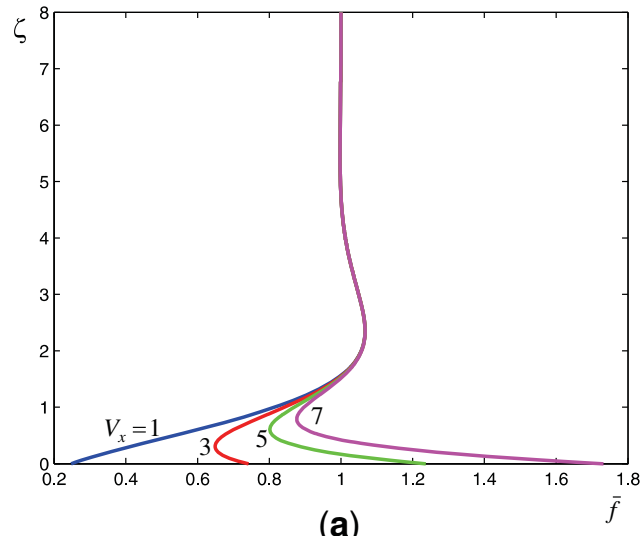

(a)

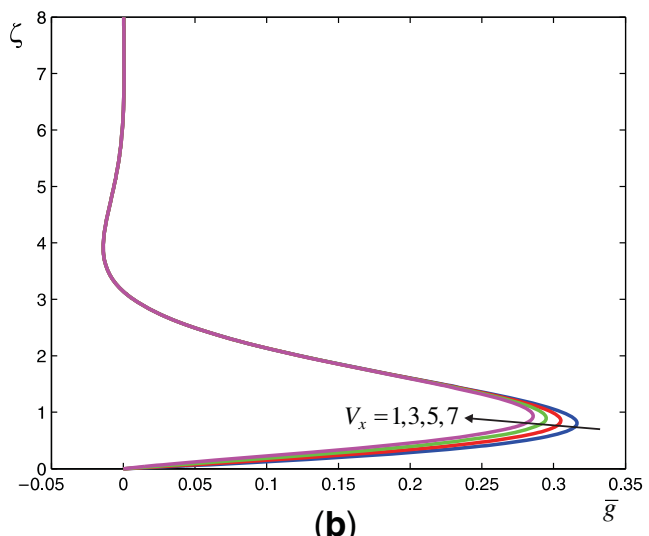

(b)

Figure 7. Variation of $\bar{f}$ and $\bar{g}$ with $\zeta$ for $k=0.5, \tau=0.5, V_{y}=0\left(V_{x}=1,3,5,7\right)$.

$$
\tilde{F}_{2}^{\prime \prime}-2 i \tilde{F}_{2}+2 k \tilde{F}_{1}=0
$$

with

$$
\begin{aligned}
\tilde{F}_{0}(0) & =-1, & \tilde{F}_{1}(0)=0, & \tilde{F}_{2}(0)=V_{x}+i V_{y}, \\
\tilde{F}_{0}(\infty) & =0, & \tilde{F}_{1}(\infty)=0, & \tilde{F}_{2}(\infty)=0 .
\end{aligned}
$$

The solutions of Eqs. (27)-(29) subject to Eq. (30) give $\bar{f}(\zeta, \tau)+i \bar{g}(\zeta, \tau)=1-\exp [-(1+i) \zeta]$

$$
\begin{aligned}
& -\frac{1}{2}\left(V_{y}-i V_{x}\right)\{\exp [M(\zeta, \tau)] \\
& -\exp [N(\zeta, \tau)]\}
\end{aligned}
$$




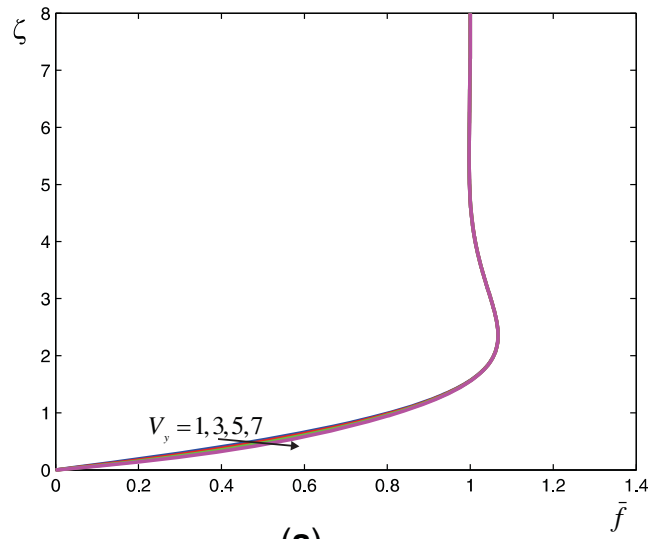

(a)

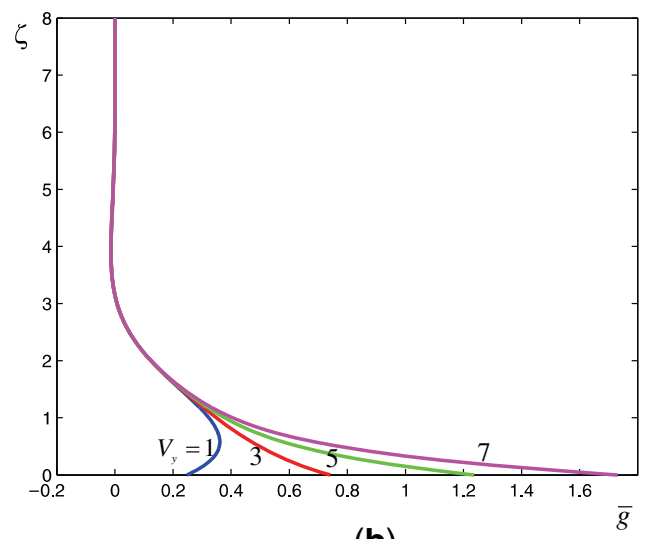

(b)

Figure 8. Variation of $\bar{f}$ and $\bar{g}$ with $\zeta$ for $k=0.5, \tau=0.5, V_{x}=0\left(V_{y}=1,3,5,7\right)$.

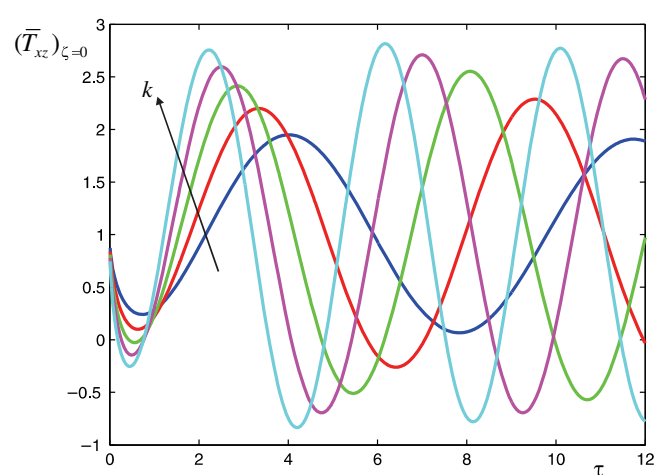

(a)

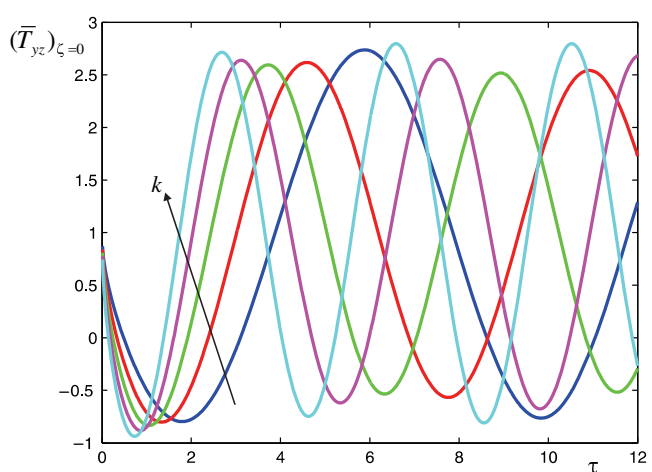

(b)

Figure 9. Variation of $\left(\bar{T}_{x z}\right)_{\zeta=0}$ and $\left(\bar{T}_{y z}\right)_{\zeta=0}$ with $\tau$ for $V_{x}=1, V_{y}=1(k=0.8,1,1.2,1.4,1.6)$.

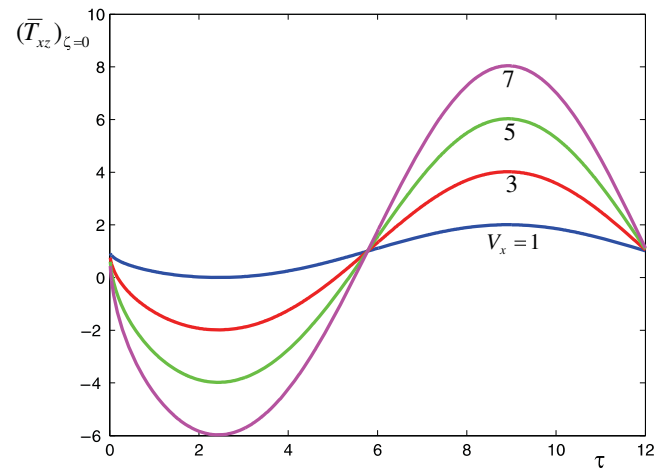

(a)

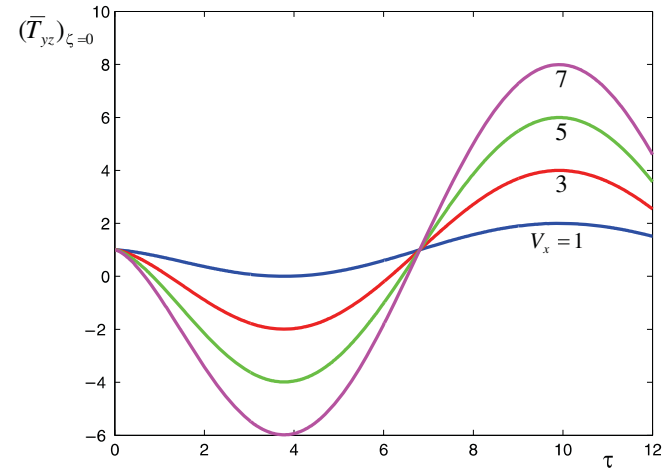

(b)

Figure 10. Variation of $\left(\bar{T}_{x z}\right)_{\zeta=0}$ and $\left(\bar{T}_{y z}\right)_{\zeta=0}$ with $\tau$ for $k=0.5, V_{y}=0\left(V_{x}=1,3,5,7\right)$. 


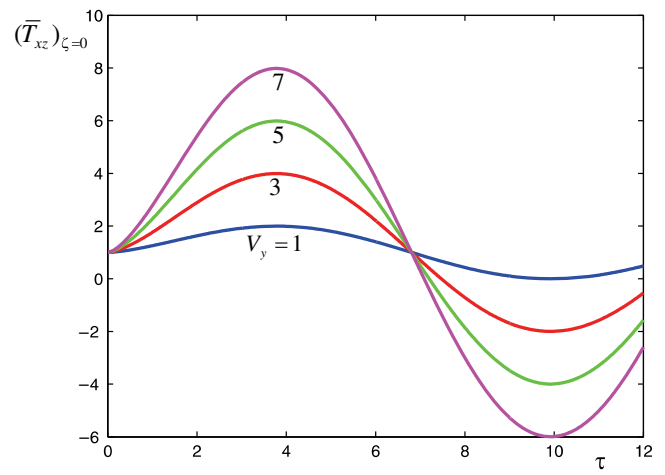

(a)

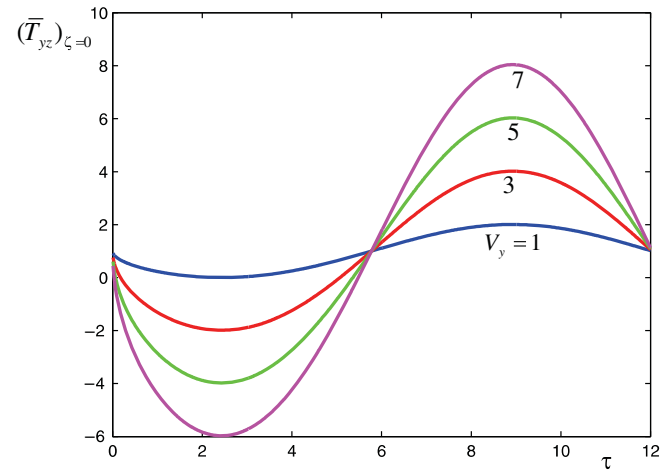

(b)

Figure 11. Variation of $\left(\bar{T}_{x z}\right)_{\zeta=0}$ and $\left(\bar{T}_{y z}\right)_{\zeta=0}$ with $\tau$ for $k=0.5, V_{x}=0\left(V_{y}=1,3,5,7\right)$.

and

$$
\begin{aligned}
\bar{T}_{x z}(\zeta, \tau)+i \bar{T}_{y z}(\zeta, \tau)=(1+i) \exp [-(1+i) \zeta] \\
-\frac{1}{2}\left(V_{x}+i V_{y}\right)\{(1+i) \sqrt{k-1} \exp [M(\zeta, \tau)] \\
+(1-i) \sqrt{k+1} \exp [N(\zeta, \tau)]\},
\end{aligned}
$$

where

$$
\begin{gathered}
M(\zeta, \tau)=(-1+i) \sqrt{k-1} \zeta-i k \tau \\
\quad N(\zeta, \tau)=-(1+i) \sqrt{k+1} \zeta+i k \tau .
\end{gathered}
$$

The solutions presented by Eqs. (31)-(33) reflect the periodic flow that occurs in the fluid. Figures 2 and 3 are plotted to observe the time when the periodic flow starts. As expected, it is observed from figures 2 and 3 that the results obtained by the periodic solution are not valid for small values of time. When the oscillation motion is set up, the flow contains transients for small times. After the initial transients decay, the motion of the fluid starts to be periodic in time. Figure 2 shows that the periodic flow starts after $\tau=8 \pi$ for $k=0.5\left(V_{x}=1, V_{y}=1\right)$ with an acceptable accuracy. On the other hand, it is concluded from figure 3 that the flow starts to be periodic after $\tau=32 \pi$ for $k=1.25\left(V_{x}=1, V_{y}=1\right)$ with sufficiently high accuracy. We infer from these figures that the time needed for the periodic flow gets larger when the ratio of the frequency of oscillation to the angular velocity of the disk increases.

\section{Results and discussion}

This paper deals with the unsteady flow of a disk executing non-torsional oscillation in its own plane and a Newtonian fluid at infinity while they are initially rotating with the same angular velocity about two parallel axes normal to the disk. The effects of all the parameters acting on the flow are studied for both the velocity field and the shear stresses that represent the components of the horizontal force per unit area exerted by the disk on the fluid. The direction of the force exerted by the fluid on the disk is opposite to that exerted by the disk on the fluid. Figures 4, 5, 6 show the variations in the $x$ - and $y$-components of the dimensionless translational velocity with time for the three cases when the ratio of the frequency of oscillation to the angular velocity of the disk is less than $1(k=0.5)$, equal to $1(k=1)$ and greater than $1(k=1.25)$. The effects of the dimensionless velocity amplitudes in the $x$ - and $y$-directions on the $x$ - and $y$-components of the dimensionless translational velocity are displayed in figures 7 and 8. The variations of the dimensionless shear stresses $\bar{T}_{x z}$ and $\bar{T}_{y z}$ at $\zeta=0$ with time are shown in figures 9, 10,11 for various values of the ratio of the frequency of oscillation to the angular velocity of the disk and the dimensionless velocity amplitudes in the $x$ and $y$-directions.

In this problem, the disk and the fluid at infinity are initially rotating about distinct axes. Hence, the initial condition is different from that in the other papers related to this flow. Further, the oscillation velocity of the disk has two components, i.e., the disk performs non-torsional oscillation both in the eccentricity direction and in the direction perpendicular to the eccentricity. Accordingly, a more general solution for the problem is obtained.

An exact solution obtained for the problem is compared with a periodic solution presented in order to observe the time when the periodic flow starts. A very good agreement is found between the two solutions for large times.

\section{Conclusions}

The main findings of the presented analysis are given below.

- The time needed for the periodic flow increases with an increase in the value of the ratio of the frequency of oscillation to the angular velocity of the disk. After the 
periodic flow occurs, the motion recurs with a period of $\tau=2 \pi / k$.

- When the oscillation occurs along the eccentricity direction, the change in the $y$-component of the translational velocity is considerably appreciable but the change in the $x$-component is almost insignificant. However, when the disk is forced to oscillate in the direction perpendicular to the eccentricity, the change in the $x$-component of the translational velocity is obviously noticed but the change in the $y$-component is much smaller.

- It is observed that there exists a phase lag between the flow velocity and the disk oscillation when the periodic flow takes place.

- The change in the $x$-component of the force per unit area exerted by the fluid on the disk gets larger when the frequency of oscillation increases. On the other hand, the change in the $y$-component gets smaller for $0<k<1$ but gets larger for $1<k$.

- The increases in the dimensionless velocity amplitudes of oscillation cause larger changes in the magnitudes of the $x$ - and $y$-components of force per unit area exerted by the fluid on the disk. Further, an interesting result is that the $x$ - and $y$-components of the force vary in an almost opposite direction when the disk executes nontorsional oscillation in the eccentricity direction. On the other hand, both the components vary in the almost same direction when the non-torsional oscillation is in the direction perpendicular to the eccentricity.

\section{Acknowledgements}

The author would like to express his sincere thanks to the referees for their valuable comments and suggestions.

\section{References}

[1] Coirier J 1972 Rotations non coaxiales d'un disque et d'un fluide à l'infini. J. Méc. 11: 317-340

[2] Kasiviswanathan S R and Rao A R 1987 An unsteady flow due to eccentrically rotating porous disk and a fluid at infinity. Int. J. Eng. Sci. 25: 1419-1425

[3] Hayat T, Asghar S and Siddiqui A M 1999 Unsteady flow of an oscillating porous disk and a fluid at infinity. Meccanica 34: 259-265

[4] Guria M, Das B K and Jana R N 2007 Oscillatory flow due to eccentrically rotating porous disk and a fluid at infinity. Meccanica 42: 487-493

[5] Erdoğan M E 2000 Flow induced by non-coaxial rotation of a disk executing non-torsional oscillations and a fluid rotating at infinity. Int. J. Eng. Sci. 38: 175-196

[6] Hayat T, Zamurad M, Asghar S and Siddiqui A M 2003 Magnetohydrodynamic flow due to non-coaxial rotations of a porous oscillating disk and a fluid at infinity. Int. J. Eng. Sci. 41: 1177-1196

[7] Hayat T, Ellahi R and Asghar S 2004a Unsteady periodic flows of a magnetohydrodynamic fluid due to noncoaxial rotations of a porous disk and a fluid at infinity. Math. Comput. Model. 40: 173-179

[8] Hayat T, Ellahi R and Asghar S 2008 Hall effects on unsteady flow due to non-coaxially rotating disk and a fluid at infinity. Chem. Eng. Commun. 195: 958-976

[9] Hayat T, Ellahi R and Asghar S 2004b Flow induced by noncoaxial rotation of a porous disk executing non-torsional oscillations and a second grade fluid rotating at infinity. Appl. Math. Model. 28: 591-605

[10] Hayat T, Ellahi R and Asghar S 2007 Unsteady magnetohydrodynamic non-Newtonian flow due to non-coaxial rotations of disk and a fluid at infinity. Chem. Eng. Commun. 194: $37-49$

[11] Wang Y and Wu W 2007 Time-dependent magnetohydrodynamic flow induced by non-coaxial rotations of a nontorsionally oscillating porous plate and a third-order fluid at infinity. Math. Comput. Model. 46: 1277-1293 